CLINICAL STUDY

\title{
Total adiponectin and adiponectin multimeric complexes in relation to weight loss-induced improvements in insulin sensitivity in obese women: the NUGENOB study
}

\author{
J Polak $^{1,2}$, Z Kovacova ${ }^{1,2}$, C Holst $^{3}$, C Verdich ${ }^{3}$, A Astrup ${ }^{4}$, E Blaak ${ }^{5}$, K Patel ${ }^{6}$, J M Oppert ${ }^{7}$, D Langin ${ }^{8,10}$, \\ J A Martinez ${ }^{9}$, T I A Sørensen ${ }^{3}$ and V Stich ${ }^{1,10}$ \\ ${ }^{1}$ Department of Sport Medicine, Third Faculty of Medicine, Charles University in Prague, 100 o0 Prague, Czech Republic, ${ }^{2}$ Division of Cell and Molecular \\ Biology, Third Faculty of Medicine, Center of Biomedical Sciences, Charles University in Prague, 100 O0 Prague, Czech Republic, ${ }^{3}$ Institute of Preventive \\ Medicine, Copenhagen University Hospital, DK-1357 Copenhagen, Denmark, ${ }^{4}$ Department of Human Nutrition, Faculty of Life Sciences, University of \\ Copenhagen, DK-1958 Copenhagen, Denmark, ${ }^{5}$ Department of Human Biology, Nutrition and Toxicology Research Institute, Maastricht University, \\ 6200 MD Maastricht, The Netherlands, ${ }^{6}$ School of Biomedical Sciences, Queen's Medical Centre, University of Nottingham Medical School, Nottingham, \\ NG7 2UH UK, ${ }^{7}$ Department of Nutrition, Hotel-Dieu Hospital, University Pierre-et-Marie Curie, F-75006 Paris, France, ${ }^{8}$ Obesity Research Unit, \\ INSERM U586, Louis Bugnard Institute and Clinical Investigation Centre, Toulouse University Hospitals, Paul Sabatier University, F-31432 Toulouse, \\ France, ${ }^{9}$ Department of Physiology and Nutrition, University of Navarra, ES-31008 Pamplona, Spain and ${ }^{10}$ Franco-Czech Laboratory for a Clinical \\ Research on Obesity, Charles University and INSERM, 10000 Prague, Czech Republic
}

(Correspondence should be addressed to J Polak who is now at 3 Lekarska Fakulta University Karlovy, Ustav Telovychovneho Lekarstvi CPL, Ruska 87, Prague 10, 100 00, Czech Republic; Email: jan.polak@lf3.cuni.cz)

\begin{abstract}
Aim: Adiponectin increases insulin sensitivity, protects arterial walls against atherosclerosis, and regulates glucose metabolism, and is decreased in obese, insulin resistant, and type 2 diabetic patients. Adiponectin circulates in plasma as high, medium, and low molecular weight forms (HMW, MMW, and LMW). The HMW form was suggested to be closely associated with insulin sensitivity. This study investigated whether diet-induced changes in insulin sensitivity were associated with changes in adiponectin multimeric complexes.

Subjects: Twenty obese women with highest and twenty obese women with lowest diet induced changes in insulin sensitivity (responders and non-responders respectively), matched for weight loss (body mass index $(\mathrm{BMI})=34.5$ (s.D. 2.9) resp. $36.5 \mathrm{~kg} / \mathrm{m}^{2}$ (s.D. 4.0) for responders and non-responders), were selected from 292 women who underwent a 10-week low-caloric diet (LCD; $600 \mathrm{kcal} / \mathrm{d}$ less than energy requirements). Plasma HMW, MMW, and LMW forms of adiponectin were quantified using Western blot method.

Results: LCD induced comparable weight reduction in responders and non-responders by 8.2 and $7.6 \mathrm{~kg}$. Homeostasis model assessment insulin resistance index decreased by $48.1 \%$ in responders and remained unchanged in non-responders. Total plasma adiponectin and the quantity of HMW and MMW remained unchanged in both groups, while LMW increased by $16.3 \%$ in non-responders. No differences between both groups were observed at baseline and after the study. Total plasma adiponectin, MMW, and LMW were negatively associated with fasting insulin levels at baseline.

Conclusion: No differences in total plasma adiponectin, HMW, MMW, and LMW forms were observed between responders and non-responders following 10-week LCD, suggesting that adiponectin is not a major determinant of weight loss-induced improvements in insulin sensitivity.
\end{abstract}

European Journal of Endocrinology 158 533-541

\section{Introduction}

Obesity is often associated with whole-body low-grade pro-inflammatory state, insulin resistance and a number of metabolic disturbances together defined as the metabolic syndrome. In the search for mechanisms that help explain the link between obesity, insulin resistance, and metabolic complications, it has been suggested that endocrine substances produced by adipose tissue (adipocytokines) might play a role (1-3). Adiponectin is a $30 \mathrm{kDa}$ plasma protein secreted by mature adipocytes (4) representing $0.01 \%$ of total plasma proteins (5). Plasma adiponectin levels have been shown to be reduced in type 2 diabetic patients (6), insulin-resistant subjects (7), obese individuals (5), as well as in coronary heart disease patients (6). These findings suggest that adiponectin has insulin-sensitizing and anti-atherosclerotic effects (8). In human plasma, adiponectin circulates in distinct multimeric complexes forming trimeric low molecular weight (LMW), hexameric medium molecular weight (MMW), and oligomeric high molecular weight (HMW) complexes. 
However, using different analytical methods, some authors distinguished only two adiponectin isoforms: LMW and HMW (7, 9, 10, 11, 12, 2). Adiponectin cell surface receptors (AdipoR1, AdipoR2) are expressed in muscle, liver, and adipose tissue $(13,14)$. In mouse myocytes and hepatocytes, adiponectin stimulates phosphorylation and the activation of $5^{\prime}$-AMP-activated protein kinase (15), a key regulatory enzyme in glucose and lipid metabolism, inducing glucose uptake and fatty acid oxidation in muscle $(16,15)$ and reducing hepatic gluconeogenesis (17). Activation of different cellular transduction pathways seems to be specific for different multimeric complexes in different organs $(18,19)$.

Previous investigations revealed that plasma HMW adiponectin levels are positively associated with insulin sensitivity indices $(11,20,12,21,22)$ and plasma highdensity lipoprotein cholesterol (HDL C), while negatively associated with body mass index (BMI) and central body fat mass (11). Mutations in the adiponectin gene associated with impaired formation of HMW complexes have been phenotypically connected with hypoadiponectinemia and type 2 diabetes in humans (19).

Low-calorie diet-induced weight loss (supported with physical activity in some studies) is the corner stone of the management of obese patients and is associated with improved insulin sensitivity (23-25). In this context, the role of distribution of adiponectin multimeric complexes has attracted interest. Recently published studies of changes in adiponectin multimeric complexes following dietary intervention have yielded contradictory results; some showing no changes in their distribution (26), while others found increased quantities of HMW, MMW, and LMW complexes $(9,27)$. These conflicting results might be partially attributable to small sample sizes and/or the inclusion of both men and women in the above-mentioned studies, as gender differences in total plasma adiponectin and in the quantity of multimeric complexes have been demonstrated (6). In fact, some previous studies reported specific association between the HMW form and the insulin sensitivity $(9,28)$, while others failed to show such a relationship (26).

The aim of this study was to investigate whether changes in insulin sensitivity promoted by diet-induced weight reduction were associated with parallel changes in quantities of adiponectin multimeric complexes. Plasma levels of adiponectin multimeric complexes were compared in two groups of obese women who were selected based on contrasting responses of insulin sensitivity to weight reduction.

\section{Materials and methods}

\section{Subjects}

A group of 292 female obese subjects participating in the low-fat diet arm of the NUGENOB study $(29,30)$ were used for subsequent selection of 'responders' and 'non-responders' in this study, as described below. During the intervention 41 women were dropped out. Only subjects fulfilling the following inclusion criteria were found eligible for this study: Caucasian race, $\mathrm{BMI}=30-40 \mathrm{~kg} / \mathrm{m}^{2}$, age $20-50$ years, free from any chronic medication treatment (including oral contraceptives), stable body weight for 3 months prior to the study beginning, normal fasting plasma glucose $(<126 \mathrm{mg} / \mathrm{dl})$, and insulin $(<2.3 \mathrm{pmol} / \mathrm{l})$ levels. From the eligible 54 subjects, both groups of responders and non-responders were randomly selected from upper (responders) or lower (non-responders) half of this group divided by changes in homeostasis model assessment insulin resistance index (HOMA-IR). A group of 20 women with the highest reduction in HOMA-IR (31) index (range: 0.85-2.30) are referred to as 'responders', a group of 20 women with the lowest reduction in HOMA-IR index (range: -0.25 to 0.53 ) are referred to as 'non-responders' in this study.

Control non-obese subjects were randomly selected from a group of 85 control female subjects enrolled in the NUGENOB study. The eligibility criteria were: Caucasian race, BMI $18.5-25 \mathrm{~kg} / \mathrm{m}^{2}$, age $20-50$ years, free from any chronic medication treatment (including oral contraceptives), stable body weight for 3 months prior to the study beginning, and normal fasting plasma glucose level $(<126 \mathrm{mg} / \mathrm{dl})$.

Subjects were recruited through the media, from waiting lists, by self referral or referral from a general physician or other clinical units and local obesity organizations in the United Kingdom (Nottingham), the Netherlands (Maastricht), France (Paris and Toulouse), Spain (Pamplona), Czech Republic (Prague), Sweden (Stockholm), and Denmark (Copenhagen).

The study protocol was approved by the Ethics Committee of each center and all subjects gave written informed consent before participating in the study. The NUGENOB trial is registered at www.controlledtrials.com under the number ISRCTN25867281.

\section{Diet intervention}

The target macronutrient composition of the diet was: $20-25 \%$ of total energy from fat, $15 \%$ from protein, and $60-65 \%$ from carbohydrate. The diet was designed to provide $600 \mathrm{kcal} / \mathrm{d}$ less than the individually estimated energy requirement, which was based on pre-treatment resting metabolic rate multiplied by a physical activity level coefficient of 1.3, assuming a sedentary life style. The dietary program is described in detail on the website http://www.nugenob.org and in a previously published paper (36). Obese women involved in this study underwent a 10-week low-caloric low-fat diet intervention. Anthropometric measurements, resting energy expenditure measurements, a standardized clinical investigation, as well as plasma samples for later analysis (stored at $-80^{\circ} \mathrm{C}$ ) were obtained at the beginning (not longer than 14 days before the beginning of the dietary counseling) 
and at the end of the tenth week of the study protocol. Participants were requested to abstain from alcohol consumption. The dietary instructions were reinforced and monitored and participants were weighed weekly. Participants were advised to follow their habitual physical activity patterns throughout the dietary intervention period. A 3-day weighed food record representing 2 weekdays and 1 weekend day was obtained before the intervention started and during the last week of the intervention. One day food records were obtained and analyzed by a dietician during the regular follow-up counseling visits in the second, fifth and seventh week. The dietary records were analyzed using the country-specific food nutrient database routinely used in each center.

\section{Anthropometric and biochemical analysis}

Body height was measured with a calibrated stadiometer. Fat mass and fat-free mass were assessed by multifrequency bioimpedance technique (Bodystat; QuadScan 4000, Isle of Man, British Isles). All blood analyses (except adiponectin and adiponectin multimeric complexes) were conducted independently of the allocated intervention groups in core facilities at the Department of Human Biology, Nutrition Research Centre NUTRIM, Maastricht University, and Medical Laboratories (Dr Stein and colleagues, Monchengladbach, Germany). Plasma glucose and lipid concentrations were measured with standard enzymatic techniques on a COBAS FARA centrifugal spectrophotometer (Roche Diagnostica), glucose (HK 125, ABX Diagnostics, Montpellier, France), triglycerides (TG; Sigma) total cholesterol (cholesterol 100, ABX Diagnostics), HDL C (Roche). Fasting plasma lowdensity lipoprotein cholesterol (LDL C) was calculated using the formula of Friedewald et al. $\left(\mathrm{LDL}_{\text {cholesterol }}=\right.$ $\left(\right.$ Total $_{\text {cholesterol }}-\mathrm{HDL}_{\text {cholesterol }}-(\mathrm{TG} / 5)$ ) (32). Fasting plasma insulin concentration was measured with a double-antibody RIA (Insulin, RIA 100, KabiPharmacia, Uppsala, Sweden). Total plasma adiponectin concentration was measured using an Adiponectin Human ELISA kit (Biovendor Laboratory Medicine Inc., Modrice, Czech Republic; coefficient of variability 4.1\%).

\section{Adiponectin multimeric complexes quantification}

Samples $(10 \mu \mathrm{l})$ of plasma diluted $(1: 2)$ with Laemmli sample buffer (without $\beta$-mercaptoethanol and SDS) were resolved using native PAGE under non-reducing, non-denaturing conditions, as previously described in other studies $(33,27,19)$. Proteins were then western blotted to a nitrocellulose membrane, blocked for $1 \mathrm{~h}$ with 5\% low-fat milk in a PBS with $0.5 \%$ Tween (PBS-T) and incubated overnight with primary human adiponectin antibody diluted 1:1000 in 1\% low-fat milk in PBS-T (Anti-Human Adiponectin Rabbit Polyclonal Antibody, Biovendor Laboratory Medicine Inc). A second incubation (45 min) was carried out with secondary antibody (goat anti-rabbit immunoglobinG conjugated with horseradish peroxidase, Jackson ImmunoResearch Europe Ltd, Cambridge, UK) diluted 1:10 000 in 1\% low-fat milk in PBS-T. Band detection using a chemiluminescent substrate (Luminol, SigmaAldrich) (34) was done on a FujiFilm LAS 1000 detection system (Fuji Photo Film Co. Ltd, Tokyo, Japan). Band intensities were analyzed using AIDA Software (Raytest, Isotopenmessgeraete $\mathrm{GmbH}$, Berlin, Germany). Plasma samples taken before and after the intervention were run on the same gel in duplicates. Signal intensities from the duplicate samples were averaged and used for statistical analysis. Native molecular weight standards (Protein Markers for Native PAGE, Serva, Heidelberg, Germany) and recombinant adiponectin (Adiponectin Human-HEK, Biovendor Laboratory Medicine Inc.) were also run on each gel. The individual signal intensity of each band was normalized using the intensity of the MMW form of the recombinant adiponectin protein. The standard was run using the identical concentration on all the gels. Coefficient of variability of the western blot analysis was $7.5 \%$. We are aware that all western blot analyses have a semi-quantitative character. However, before versus after comparisons are meaningful, assuming plasma samples derived before and after the intervention are run on the same gel. Consequently western blots provide a useful analytical tool for analyses of all adiponectin multimeric complexes in human plasma, as has been demonstrated by other studies $(26,27)$.

\section{Statistical analysis}

Statistical analysis was performed using SPSS 12.0 for Windows (SPSS Inc., Chicago, IL, USA). The effect of intervention was tested using the Wilcoxon test for paired observations for all studied variables. Differences in the effect of dietary intervention between responders and non-responders were analyzed using two-sample Mann-Whitney test. Comparison of baseline values between controls and groups undergoing intervention was done using two-sample Mann-Whitney test. Univariate correlations were analyzed using the Pearson's correlation test with or without controlling for fat mass. As no differences in anthropometrical or biochemical data were observed between responders and non-responders at baseline, values of both groups were pooled together for the analysis of baseline associations. The HOMA-IR index was computed following the equation: ((fasting glucose $\mathrm{(mmol} / \mathrm{l}) \times$ fasting $\left.\left.\operatorname{insulin}_{(\mathrm{uIU} / \mathrm{ml})}\right) / 22.5\right)$. Data are presented as mean \pm s.D. A level of $P \leq 0.05$ was considered statistically significant in all tests. 
Table 1 Energy intake and macronutrient intake in responders and non-responders before and at the end of a 10-week dietary intervention.

\begin{tabular}{|c|c|c|c|c|c|}
\hline & \multicolumn{2}{|c|}{ Responders $(n=20)$} & \multicolumn{2}{|c|}{ Non-responders $(n=20)$} & \multirow{2}{*}{$\begin{array}{c}\text { Responders versus } \\
\text { non-responders } \\
P \text { value }\end{array}$} \\
\hline & Baseline & After LCD & Baseline & After LCD & \\
\hline Energy intake (kcal/day) & $1897.4 \pm 549.8$ & $1371.1 \pm 297.5^{\star}$ & $1942.4 \pm 384.6$ & $1456.9 \pm 204.4^{*}$ & 0.792 \\
\hline Fat energy $(\%)$ & $36.4 \pm 6.7$ & $24.4 \pm 4.5^{\star}$ & $36.7 \pm 7.8$ & $23.7 \pm 4.1^{*}$ & 0.857 \\
\hline Carbohydrate energy (\%) & $45.4 \pm 9.2$ & $57.0 \pm 4.6^{*}$ & $45.8 \pm 8.5$ & $57.1 \pm 5.5^{\star}$ & 0.901 \\
\hline Protein energy (\%) & $16.9 \pm 6.0$ & $18.1 \pm 1.8$ & $16.7 \pm 3.6$ & $19.0 \pm 2.6^{\dagger}$ & 0.687 \\
\hline
\end{tabular}

Data are presented as mean \pm S.D. . ${ }^{*} P<0.001$ and ${ }^{\dagger} P<0.05$ for paired comparisons before versus after $L C D$ intervention (Wilcoxon test). Responders versus non-responders column shows $P$ values of Mann-Whitney test comparing the differences in the changes of variables between responders and non-responders.

\section{Results}

\section{Dietary intervention and changes in anthropometric and biochemical parameters}

The energy intake and macronutrient composition of the diet before the beginning of the study and during the last week of the intervention are summarized in Table 1, indicating that diet composition changed from baseline (the effect of the intervention), but no differences in the diet composition were observed between the two groups at baseline or at the end of the intervention.

Anthropometric and biochemical characteristics of the subjects before and after the dietary intervention are summarized in Table 2. Ten weeks of low-calorie diet (LCD) resulted in a reduction of body weight, BMI, fat mass, and waist circumference by 8.1, 8.2, 13.7, and $6.4 \%$ respectively in obese women (responders and nonresponders grouped together). No significant differences in baseline or diet-induced changes of these parameters were observed between responders and non-responders.
There were no differences in baseline or diet-induced changes in metabolic and hormonal variables between the two groups, except diet-induced decreases in plasma insulin levels that were higher in responders compared with non-responders as designed and plasma TG, which were reduced in responders but remained unchanged in non-responders. Leptin levels significantly decreased following the LCD in responders as well as nonresponders with no differences between both groups. HOMA-IR decreased in responders by $48.1 \%(P<0.05)$ and in non-responders by $6.3 \%(P<0.05)$ following LCD (according to the selection criteria for responders and non-responders).

\section{Total plasma adiponectin and adiponectin multimeric complexes}

Total plasma adiponectin levels were not different between responders and non-responders at baseline and at the end of the study. Compared with obese

Table 2 Anthropometric and biochemical variables in control subjects, responders, and non-responders before and at the end of 10-week dietary intervention.

\begin{tabular}{|c|c|c|c|c|c|c|}
\hline & \multicolumn{2}{|c|}{ Responders $(n=20)$} & \multicolumn{2}{|c|}{ Non-responders $(n=20)$} & \multirow[b]{2}{*}{$\begin{array}{c}\text { Controls } \\
(n=20)\end{array}$} & \multirow{2}{*}{$\begin{array}{c}\text { Responders versus } \\
\text { non-responders } \\
P \text { value }\end{array}$} \\
\hline & Baseline & After LCD & Baseline & After LCD & & \\
\hline Weight (kg) & $94.0 \pm 12.9$ & $85.8 \pm 13.3^{*}$ & $97.2 \pm 10.8$ & $89.6 \pm 11.4^{*}$ & $60.1 \pm 5.6^{\ddagger, \S}$ & 0.365 \\
\hline BMI $\left(\mathrm{kg} / \mathrm{m}^{2}\right)$ & $34.5 \pm 2.9$ & $31.5 \pm 3.5^{*}$ & $36.5 \pm 4.0$ & $33.7 \pm 4.3^{*}$ & $21.3 \pm 1.5^{\ddagger, \S}$ & 0.369 \\
\hline Waist $(\mathrm{cm})$ & $101.3 \pm 7.8$ & $94.0 \pm 8.2^{*}$ & $105.5 \pm 11.5$ & $99.6 \pm 10.9^{*}$ & $70.0 \pm 6.1^{ \pm, \S}$ & 0.331 \\
\hline Fat mass \% & $40.4 \pm 7.3$ & $34.2 \pm 8.6^{*}$ & $44.4 \pm 8.0$ & $38.8 \pm 8.6^{\star}$ & $14.8 \pm 3.0^{\ddagger, \S}$ & 0.339 \\
\hline Fat-free mass $\%$ & $53.6 \pm 6.7$ & $51.3 \pm 6.0^{*}$ & $52.7 \pm 4.1$ & $51.1 \pm 4.6^{*}$ & $45.4 \pm 4.6^{\ddagger, \S}$ & 0.603 \\
\hline Glucose (mmol/l) & $5.3 \pm 0.3$ & $5.1 \pm 0.3^{\dagger}$ & $5.3 \pm 0.4$ & $5.3 \pm 0.5$ & $4.9 \pm 0.4^{\ddagger, \S}$ & 0.175 \\
\hline Insulin (mU/I) & $11.3 \pm 2.9$ & $6.0 \pm 3.1^{*}$ & $10.0 \pm 2.7$ & $9.4 \pm 2.7^{\dagger}$ & $4.3 \pm 2.8^{\ddagger, \S}$ & 0.000 \\
\hline HOMA-IR & $2.7 \pm 0.7$ & $1.4 \pm 0.8^{\dagger}$ & $2.4 \pm 0.8$ & $2.2 \pm 0.8^{\dagger}$ & $0.9 \pm 0.6^{\ddagger, \S}$ & 0.000 \\
\hline Cholesterol $(\mathrm{mmol} / \mathrm{l})$ & $5.4 \pm 0.8$ & $4.8 \pm 0.6^{\dagger}$ & $4.8 \pm 1.0$ & $4.5 \pm 1.0^{\dagger}$ & $4.3 \pm 0.8^{\ddagger}$ & 0.247 \\
\hline $\mathrm{HDL} \mathrm{C}(\mathrm{mmol} / \mathrm{l})$ & $1.1 \pm 0.2$ & $0.9 \pm 0.2^{*}$ & $1.0 \pm 0.2$ & $0.9 \pm 0.1$ & $1.4 \pm 0.3^{\ddagger, \S}$ & 0.127 \\
\hline LDL C (mmol/l/) & $3.7 \pm 0.8$ & $3.4 \pm 0.6^{\dagger}$ & $3.3 \pm 0.5$ & $3.1 \pm 0.9^{\dagger}$ & $2.7 \pm 0.7^{\ddagger \S \S}$ & 0.771 \\
\hline Triglycerides $(\mathrm{mmol} / \mathrm{l})$ & $1.2 \pm 0.4$ & $1.0 \pm 0.3^{\dagger}$ & $1.0 \pm 0.4$ & $1.0 \pm 0.4$ & $0.6 \pm 0.2^{\ddagger \S \S}$ & 0.04 \\
\hline Leptin (ng/ml) & $25.7 \pm 10.3$ & $16.4 \pm 10.1^{*}$ & $27.1 \pm 8.4$ & $21.1 \pm 9.9^{*}$ & $8.6 \pm 4.0^{\ddagger \S}$ & 0.176 \\
\hline
\end{tabular}

Data are presented as mean \pm s.D.. BMI, body mass index; HOMA-IR, homeostasis model assessment insulin resistance index; HDL C, high-density lipoprotein cholesterol; LDL C, low-density lipoprotein cholesterol; LCD, low-calorie diet. ${ }^{\star} P<0.001$ and ${ }^{\dagger} P<0.05$ for paired comparisons before versus after diet intervention (Wilcoxon test). ${ }^{\ddagger} P<0.01$ for comparisons of baseline values between responders and controls (Mann-Whitney test). ${ }^{\S} P<0.01$ for comparisons of baseline values between non-responders and controls (Mann-Whitney test). Responders versus non-responders column shows $P$ values of Mann-Whitney test comparing the differences in the changes of variables between responders and non-responders. 
subjects (responders and non-responders together), control lean individuals had higher plasma levels of total adiponectin by $19.3 \%(P=0.05)$. Diet intervention did not induce changes in total plasma adiponectin levels either in responders or in non-responders.

The quantity of the HMW, MMW, and LMW forms was not different between the responders and nonresponders at baseline or at the end of the study. Control lean subjects showed higher levels of the MMW and LMW forms by 84.3 and $45.2 \%$ (both $P<0.05$ ) compared with non-responders at the beginning of the study, while no significant differences were observed between responders and control subjects. Absolute quantity of the LMW form was increased by $16.3 \%$ $(P<0.05)$ following the LCD in non-responders. No other changes in the quantity of HMW or MMW forms were detected. Data are summarized in Table 3.

\section{Relationship between the total plasma adiponectin and anthropometric/metabolic variables}

In obese subjects (responders and non-responders together), total plasma adiponectin at baseline was negatively associated with waist circumference, fasting insulin levels, HOMA-IR index, and TG/HDL $C$ ratio $(r=-0.29,-0.27,-0.29$, and -0.44 respectively, all $P<0.05)$ and positively associated with HDL cholesterol $(r=0.4, P<0.01)$. When controlling for fat mass in the analyses, only association between plasma adiponectin, HDL cholesterol and TG/HDL ratio remained significant. No other associations between the total plasma adiponectin and the analyzed biochemical and anthropometrical parameters were observed.

\section{Relationships between adiponectin multimeric complexes and anthropometric/metabolic variables}

In obese subjects (responders and non-responders together), the LMW and MMW forms were negatively associated with fat mass $(r=-0.30$ and -0.36 respectively, $P<0.05)$, BMI $(r=-0.30$ and -0.34 respectively, $P<0.05)$, waist circumference $(r=-0.28$ and -0.38 respectively, $P<0.05)$, and fasting insulin $(r=-0.29$ and -0.26 respectively, $P<0.05)$ at the beginning of the study. HOMA-IR index was associated with the MMW form at the beginning of the study $(r=-0.28, P<0.05)$; an association with the LMW form was of borderline significance $(r=-0.24, P=0.07)$. These associations were not significant when controlled for fat mass or when responders and non-responders were analyzed separately. No associations between the HMW form and the anthropometrical or biochemical parameters at baseline were present. Diet-induced changes in the analyzed anthropometric and biochemical parameters were not associated with changes in the absolute or relative quantity of adiponectin multimeric complexes in responders or non-responders.

\section{Discussion}

The present study investigates for the first time in a retrospective design the diet-induced changes in insulin resistance related to plasma adiponectin and levels of adiponectin multimeric complexes. Responders and nonresponders $(2 \times 20$ subjects $)$ with respect to their HOMAIR index changes following the dietary intervention were selected from 292 women who participated in a multicentre interventional study NUGENOB. When baseline and post-intervention changes in total plasma adiponectin and its multimeric forms were compared, no significant differences were detected between the responders and the non-responders. It has to be emphasized here that this retrospective approach enabled defining unique groups of subjects, so that baseline as well as diet-induced changes of variables related to insulin sensitivity (body weight, fat mass, BMI, waist circumference, fasting plasma glucose, free fatty acids) were not statistically different between both groups, while insulin sensitivity was improved only in responders, mainly due to changes in fasting plasma insulin levels. It is thus not plausible to explain the opposite evolution of HOMA-IR index in both

Table 3 Total plasma adiponectin and the HMW, MMW and LMW forms in control subjects, responders and nonresponders before and at the end of 10 -week dietary intervention.

\begin{tabular}{|c|c|c|c|c|c|c|c|c|}
\hline & \multicolumn{3}{|c|}{ Responders $(n=20)$} & \multicolumn{3}{|c|}{ Non-responders $(n=20)$} & \multirow[b]{2}{*}{$\begin{array}{l}\text { Controls } \\
(n=20)\end{array}$} & \multirow{2}{*}{$\begin{array}{c}\text { Responders versus } \\
\text { non-responders } \\
P \text { value }\end{array}$} \\
\hline & Baseline & After LCD & Change & Baseline & After LCD & Change & & \\
\hline Adip & $7.1 \pm 2.7$ & $6.7 \pm 2.5$ & $0.3 \pm 0.9$ & $7.6 \pm 2.8$ & $7.8 \pm 2.7$ & $0.2 \pm 2.1$ & $9.1 \pm 3.7$ & 0.559 \\
\hline HMW f & $14.7 \pm 13.4$ & $15.6 \pm 14.6$ & $0.9 \pm 5.0$ & $9.9 \pm 6.6$ & $9.6 \pm 6.5$ & $0.3 \pm 1.7$ & $15.5 \pm 8.0$ & 0.273 \\
\hline MMW form (QL) & $17.1 \pm 13.8$ & $17.5 \pm 14.9$ & $2.8 \pm 8.0$ & $13.4 \pm 10.5$ & $13.6 \pm 11.8$ & $3.7 \pm 6.6$ & $24.7 \pm 12.2^{\dagger}$ & 0.911 \\
\hline LMW form (QL) & $15.5 \pm 8.9$ & $16.4 \pm 9.2$ & $1.7 \pm 10.7$ & $13.5 \pm 6.8$ & $15.7 \pm 8.9^{*}$ & $5.8 \pm 5.4$ & $19.6 \pm 7.0^{\dagger}$ & 0.249 \\
\hline
\end{tabular}

Data are presented as mean \pm s.D.; HMW, high molecular weight form; MMW, medium molecular weight form; LMW, low molecular weight form; QL, quantity of light units normalized by reference $\mathrm{QL}$ of recombinant adiponectin MMW; LCD, low calorie diet. * $P<0.05$ for paired comparisons before versus after (Wilcoxon test). ${ }^{\dagger} P<0.01$ for comparisons of baseline values between non-responders and controls (Mann-Whitney test). Responders versus non-responders column shows $P$ values of Mann-Whitney test comparing the differences in the changes of variables between responders and non-responders. 
the groups in terms of different reduction in the analyzed anthropometric variables. Despite the published associations between adiponectin levels and parameters of insulin sensitivity at baseline $(11,20,12,21,22)$, the precise position of adiponectin in diet-induced changes in insulin resistance remain undisclosed. If adiponectin plays a dominant role in weight loss-induced changes in insulin sensitivity, we would expect to observe different adiponectin plasma levels or adiponectin multimeric complex quantities between responders and non-responders. However, this hypothesis was not supported by data from the present study. Based on the results of this study, it can be concluded that adiponectin seems not to be a major determinant of the weight loss-induced changes in wholebody insulin sensitivity in women.

Responders and non-responders achieved a comparable reduction of body weight $(8.7$ and $7.8 \%)$, BMI (8.7 and $7.7 \%$ ), and fat mass (15.3 and $12.6 \%)$, suggesting a good compliance with the dietary protocol in both the groups. Plasma lipid profile parameters were also influenced as expected in both the groups. Quantity of the changes in anthropometrical variables are in the range observed in other interventional studies with comparable dietary protocols $(35,36)$ as well as those in the whole NUGENOB study (30).

The results currently available in the literature concerning changes in total plasma adiponectin concentration after weight loss are inconsistent. Unchanged levels of total plasma adiponectin following liposuction (37) or during a moderate weight loss were found in several studies $(26,38,9)$, while an increase in plasma adiponectin following extensive weight reductions by bariatric surgery $(39,28,40,41)$ or intensive lifestyle counseling (42) were described by others.

Five papers on weight loss-induced changes in plasma distribution of adiponectin multimeric complexes have been published so far (four dietary interventions, are bariatric surgery intervention), showing no changes in distribution of multimeric complexes (26), isolated increase of the HMW form (28), an increase in the HMW and MMW forms $(9,43)$, or an increase in all the multimeric forms (27). However, there are differences in the male/female proportion and methods used for the analysis of multimeric complexes among these studies that should not be overlooked. In the present study, we investigated the largest number of subjects so far, consisting of 40 subjects (responders and non-responders). Because marked differences in total plasma adiponectin and its multimeric complexes were reported between men and women (women showing higher values of total adiponectin and HMW form) (6), we decided to investigate only female subjects in this study to reduce the inter-individual variability. We observed no diet-induced changes in adiponectin multimeric complexes following diet-induced weight loss, which support the findings of Abbasi et al. (26) but contrasts to the results of Bobbert et al. (9) study. It should be emphasized here that differences exist in the methods used for native electrophoresis between laboratories and even minor differences at this crucial step might produce profound differences in multimeric complexes distribution (44).

Similar to other studies $(5,45,6,46,20,21)$, a strong negative associations between total plasma adiponectin and indices of insulin sensitivity (HOMAIR index and fasting insulin levels) were found in the present study by a univariate correlation analysis at baseline when obese subjects were investigated (responders and non-responders together). Among the analyzed multimeric complexes, a negative association between the LMW and MMW forms and baseline fasting insulin levels and between HOMA-IR index and the MMW forms were observed in our study. However, these correlations remained non-significant when controlling for body fat mass, suggesting that adiposity has an important impact on total plasma adiponectin, its multimeric complexes as well as insulin sensitivity and that associations between insulin resistance and adiponectin (total as well as multimeric isoforms) might be only reflecting the excessive adipose tissue stores. We also analyzed the association between the TG/HDL C ratio, which was recently shown to be useful in assessing insulin resistance and risk of atherosclerosis $(48,49)$, and total plasma adiponectin. TG/HDL ratio at baseline was strongly inversely associated with total plasma adiponectin level, independently on body weight, but not with any of the multimeric complexes in obese subjects (responders and non-responders pooled together). Diet-induced changes in the TG/HDL ratio were not associated with changes in plasma adiponectin levels or levels of multimeric complexes separately in responders and non-responders. These findings suggest that adiponectin might participate in a regulation of lipid metabolism at baseline. However, weight-loss induced changes in lipid metabolism seem to be induced mainly by changes in adiposity.

In agreement with the previous findings $(26,9)$, we observed neither diet-induced changes of HMW, MMW, or LMW form nor their association with changes of parameters of insulin sensitivity. The only dietresponsive form was the LMW form in a group of non-responders with $16.3 \%$ increase following the intervention, which is congruent with the results of our previous study (27), where the LMW form was predominantly up-regulated following low-calorie diet. However, this isolated statistical significance might also be a matter of chance. Another possible explanation why associations between changes in insulin sensitivity and adiponectin levels were not observed in our study is that HOMA-IR in investigated subjects was within the normal range, i.e., subjects were not insulin resistant despite being diagnosed as obese. It can be hypothesized that changes in insulin sensitivity achieved in insulinresistant populations might be more strongly associated with changes in adiponectin (and its multimeric complexes) levels. Furthermore, insulin sensitivity 
might be improved through changes in other plasma cytokines (interleukin-6, tumor necrosis factor, leptin) $(50,47)$ or a reduction in fat cell size and intrahepatic lipid content (36). Among these, leptin was analyzed in our study showing an expected decrease following intervention in both groups but no differences between both groups were observed, suggesting that neither leptin is discriminating responders from non-responders. Additionally caloric restriction per se was also shown to improve insulin sensitivity (51).

It is also possible that associations of changes in HMW, MMW, and LMW forms with changes in insulin sensitivity might be more specific to thiazolidindione (TZD) treatment than to $\operatorname{LCD}(52,43,12)$. As intervention-induced changes in HOMA-IR, fasting glucose and insulin levels were closely associated with parallel changes in fat mass, $(r=0.58,0.68$, and 0.47 , all $P<0.05)$ in responders, it can be suggested that changes in adiposity became major determinants of changes in insulin sensitivity, probably independent of adiponectin. Similar regulation was suggested during the exercise-induced weight loss in another study (53).

HOMA-IR, like any other index of insulin sensitivity based on fasting levels of insulin and glucose is evaluating mostly hepatic insulin sensitivity. As HOMA-IR does not evaluate insulin-stimulated glucose uptake, which takes place predominantly in skeletal muscle after glucose administration, any dietary intervention-induced changes in skeletal-muscle glucose uptake remained undisclosed. Although it has been shown that HOMA-IR correlates surprisingly well with the insulin sensitivity indices obtained using hyperinsulinemic clamp technique in obese individuals (54), it is necessary to note that HOMA-IR is largely dependent on fasting insulin levels. Excursions of insulin levels after several hormonal and metabolic stimuli together with a relatively short serum half-life and pulsatile secretion are responsible for the observed variability of HOMA in obese individuals, where coefficient of variability equal to 0.58 was reported compared with 0.28 in lean subjects (54). It has also been demonstrated that the association between HOMA-IR and clamp-derived indices might be diminished in lean (insulin sensitive) as well as severely insulin-resistant subjects (type 2 diabetes) (54).

The HMW form has been suggested to be physiologically the most potent form of adiponectin and might be the form responsible for its beneficial insulinsensitizing and anti-atherosclerotic effects $(43,12)$. It has been shown that the ratios of HMW/total adiponectin and HMW/LMW are plausible indicators of TZD-induced changes in insulin sensitivity (12). The quantity of the HMW form in our study was not different between obese responders and non-responders at baseline or following the LCD, while the quantity of LMW and MMW was lower in non-responders compared with lean controls at baseline. The HMW/total adiponectin ratio was also not different in responders and non- responders at baseline in our study. Our results thus do not support the hypothesis of a predominant role of the HMW form in influencing insulin sensitivity and interpretation of the HMW/total adiponectin ratio results should be performed with caution.

In conclusion, total plasma adiponectin together with the MMW and LMW forms (borderline significance) were found negatively associated with indices of insulin resistance at baseline in obese women. No differences in total plasma adiponectin or multimeric complexes were observed between subjects with the highest and lowest change of HOMA-IR index following a 10-week hypoenergetic diet. Further studies elucidating the physiological relevance and function of multimeric complexes of adiponectin with respect to obesity and insulin resistance are warranted.

\section{Acknowledgements}

This study was funded by a European Commission grant under the 5th Framework Programme project NUGENOB (EU grant QLK1-CT-2000-00618), by the grant of the Grant Agency of Charles University in Prague No. 72/2005C and by the Research Project of the Czech Ministry of Education MSM 0021620814. The NUGENOB Study and the Consortium conducting it are described on this website: www.nugenob.org.

\section{References}

1 Berg AH \& Scherer PE. Adipose tissue, inflammation, and cardiovascular disease. Circulation Research 200596 939-949.

2 Richards AA, Hickman IJ, Wang AY, Jones AL, Newell F, Mowry BJ, Whitehead JP, Prins JB \& Macdonald GA. Olanzapine treatment is associated with reduced high molecular weight adiponectin in serum: a potential mechanism for olanzapine-induced insulin resistance in patients with schizophrenia. Journal of Clinical Psychopharmacology 200626 232-237.

3 Rondinone CM. Adipocyte-derived hormones, cytokines, and mediators. Endocrine 200629 81-90.

4 Scherer PE, Williams S, Fogliano M, Baldini G \& Lodish HFM. A novel serum protein similar to $\mathrm{C} 1 \mathrm{q}$, produced exclusively in adipocytes. Journal of Biological Chemistry $199527026746-26749$.

5 Arita Y, Kihara S, Ouchi N, Takahashi M, Maeda K, Miyagawa J, Hotta K, Shimomura I, Nakamura T, Miyaoka K, Kuriyama H, Nishida M, Yamashita S, Okubo K, Matsubara K, Muraguchi M, Ohmoto Y, Funahashi T \& Matsuzawa Y. Paradoxical decrease of an adipose-specific protein, adiponectin, in obesity. Biochemical and Biophysical Research Communications 1999257 79-83.

6 Hotta K, Funahashi T, Arita Y, Takahashi M, Matsuda M, Okamoto Y, Iwahashi H, Kuriyama H, Ouchi N, Maeda K, Nishida M, Kihara S, Sakai N, Nakajima T, Hasegawa K, Muraguchi M, Ohmoto Y, Nakamura T, Yamashita S, Hanafusa T \& Matsuzawa Y. Plasma concentrations of a novel, adipose-specific protein, adiponectin, in type 2 diabetic patients. Arteriosclerosis, Thrombosis, and Vascular Biology 200020 1595-1599.

7 Abbasi F, Chu JW, Lamendola C, McLaughlin T, Hayden J, Reaven GM \& Reaven PD. Discrimination between obesity and insulin resistance in the relationship with adiponectin. Diabetes $200453585-590$.

8 Ouchi N, Kihara S, Arita Y, Nishida M, Matsuyama A, Okamoto Y, Ishigami M, Kuriyama H, Kishida K, Nishizawa H, Hotta K, 
Muraguchi M, Ohmoto Y, Yamashita S, Funahashi T \& Matsuzawa Y. Adipocyte-derived plasma protein, adiponectin, suppresses lipid accumulation and class A scavenger receptor expression in human monocyte-derived macrophages. Circulation 2001103 1057-1063.

9 Bobbert T, Rochlitz H, Wegewitz U, Akpulat S, Mai K, Weickert MO, Mohlig M, Pfeiffer AF \& Spranger J. Changes of adiponectin oligomer composition by moderate weight reduction. Diabetes $2005542712-2719$.

10 Korner A, Wabitsch M, Seidel B, Fischer-Posovszky P, Berthold A, Stumvoll M, Bluher M, Kratzsch J \& Kiess W. Adiponectin expression in humans is dependent on differentiation of adipocytes and down-regulated by humoral serum components of high molecular weight. Biochemical and Biophysical Research Communications 2005337 540-550.

11 Lara-Castro C, Luo N, Wallace P, Klein RL \& Garvey WT. Adiponectin multimeric complexes and the metabolic syndrome trait cluster. Diabetes 200655 249-259.

12 Pajvani UB, Hawkins M, Combs TP, Rajala MW, Doebber T, Berger JP, Wagner JA, Wu M, Knopps A, Xiang AH, Utzschneider KM, Kahn SE, Olefsky JM, Buchanan TA \& Scherer PE. Complex distribution, not absolute amount of adiponectin, correlates with thiazolidinedione-mediated improvement in insulin sensitivity. Journal of Biological Chemistry 2004 279 12152-12162.

$13 \mathrm{Fu} \mathrm{Y,} \mathrm{Luo} \mathrm{N,} \mathrm{Klein} \mathrm{RL} \mathrm{\&} \mathrm{Garvey} \mathrm{WT.} \mathrm{Adiponectin} \mathrm{promotes}$ adipocyte differentiation, insulin sensitivity, and lipid accumulation. Journal of Lipid Research 200546 1369-1379.

14 Yamauchi T, Kamon J, Ito Y, Tsuchida A, Yokomizo T, Kita S, Sugiyama T, Miyagishi M, Hara K, Tsunoda M, Murakami K, Ohteki T, Uchida S, Takekawa S, Waki H, Tsuno NH, Shibata Y, Terauchi Y, Froguel P, Tobe K, Koyasu S, Taira K, Kitamura T, Shimizu T, Nagai $R$ \& Kadowaki T. Cloning of adiponectin receptors that mediate antidiabetic metabolic effects. Nature 2003423 762-769.

15 Yamauchi T, Kamon J, Minokoshi Y, Ito Y, Waki H, Uchida S, Yamashita S, Noda M, Kita S, Ueki K, Eto K, Akanuma Y, Froguel P, Foufelle F, Ferre P, Carling D, Kimura S, Nagai R, Kahn BB \& Kadowaki T. Adiponectin stimulates glucose utilization and fattyacid oxidation by activating AMP-activated protein kinase. Nature Medicine 20028 1288-1295.

16 Winder WW \& Hardie DG. AMP-activated protein kinase, a metabolic master switch: possible roles in type 2 diabetes. American Journal of Physiology 1999277 E1-E10.

17 Lochhead PA, Salt IP, Walker KS, Hardie DG \& Sutherland C. 5-Aminoimidazole-4-carboxamide riboside mimics the effects of insulin on the expression of the 2 key gluconeogenic genes PEPCK and glucose-6-phosphatase. Diabetes 200049 896-903.

18 Tsao TS, Tomas E, Murrey HE, Hug C, Lee DH, Ruderman NB, Heuser JE \& Lodish HF. Role of disulfide bonds in Acrp30/adiponectin structure and signaling specificity. Different oligomers activate different signal transduction pathways. Journal of Biological Chemistry 2003278 50810-50817.

19 Waki H, Yamauchi T, Kamon J, Ito Y, Uchida S, Kita S, Hara K, Hada Y, Vasseur F, Froguel P, Kimura S, Nagai R \& Kadowaki T. Impaired multimerization of human adiponectin mutants associated with diabetes. Molecular structure and multimer formation of adiponectin. Journal of Biological Chemistry $2003 \mathbf{2 7 8}$ $40352-40363$.

20 Matsubara M, Katayose S \& Maruoka S. Decreased plasma adiponectin concentrations in nondiabetic women with elevated homeostasis model assessment ratios. European Journal of Endocrinology $2003 \mathbf{1 4 8} 343-350$.

21 Tschritter O, Fritsche A, Thamer C, Haap M, Shirkavand F, Rahe S, Staiger H, Maerker E, Haring H \& Stumvoll M. Plasma adiponectin concentrations predict insulin sensitivity of both glucose and lipid metabolism. Diabetes 200352 239-243.

22 von Eynatten M, Hamann A, Twardella D, Nawroth PP, Brenner H \& Rothenbacher D. Relationship of adiponectin with markers of systemic inflammation, atherogenic dyslipidemia, and heart failure in patients with coronary heart disease. Clinical Chemistry $200652853-859$.
23 Brook RD. Obesity, weight loss, and vascular function. Endocrine 200629 21-25.

24 Eddy DM, Schlessinger L \& Kahn R. Clinical outcomes and costeffectiveness of strategies for managing people at high risk for diabetes. Annals of Internal Medicine 2005143 251-264.

25 Knowler WC, Barrett-Connor E, Fowler SE, Hamman RF, Lachin JM, Walker EA \& Nathan DM. Reduction in the incidence of type 2 diabetes with lifestyle intervention or metformin. New England Journal of Medicine 2002346 393-403.

26 Abbasi F, Chang SA, Chu JW, Ciaraldi TP, Lamendola C, McLaughlin T, Reaven GM \& Reaven PD. Improvements in insulin resistance with weight loss, in contrast to rosiglitazone, are not associated with changes in plasma adiponectin or adiponectin multimeric complexes. American Journal of Physiology. Regulatory, Integrative and Comparative Physiology 2006290 R139-R144.

27 Polak J, Kovacova Z, Jacek M, Klimcakova E, Kovacikova M, Vitkova M, Kuda O, Sebela M, Samcova E \& Stich V. An increase in plasma adiponectin multimeric complexes follows hypocaloric diet-induced weight loss in obese and overweight premenopausal women. Clinical Science 200712 557-565.

28 Salani B, Briatore L, Andraghetti G, Adami GF, Maggi D \& Cordera R. High-molecular weight adiponectin isoforms increase after biliopancreatic diversion in obese subjects. Obesity 200614 1511-1514.

29 Petersen M, Taylor MA, Saris WH, Verdich C, Toubro S, Macdonald I, Rossner S, Stich V, Guy-Grand B, Langin D, Martinez JA, Pedersen O, Holst C, Sorensen TI \& Astrup A. Randomized, multi-center trial of two hypo-energetic diets in obese subjects: high- versus low-fat content. International Journal of Obesity 200630 552-560.

30 Sorensen TI, Boutin P, Taylor MA, Larsen LH, Verdich C, Petersen L, Holst C, Echwald SM, Dina C, Toubro S, Petersen M, Polak J, Clement K, Martinez JA, Langin D, Oppert JM, Stich V, Macdonald I, Arner P, Saris WH, Pedersen O, Astrup A \& Froguel P. Genetic polymorphisms and weight loss in obesity: a randomised trial of hypo-energetic high- versus low-fat diets. PLoS Clinical Trials 20061 e12.

31 Matthews DR, Hosker JP, Rudenski AS, Naylor BA, Treacher DF \& Turner RC. Homeostasis model assessment: insulin resistance and beta-cell function from fasting plasma glucose and insulin concentrations in man. Diabetologia 198528 412-419.

32 Friedewald WT, Levy RI \& Fredrickson DS. Estimation of the concentration of low-density lipoprotein cholesterol in plasma, without use of the preparative ultracentrifuge. Clinical Chemistry 197218 499-502.

33 Laemmli UK. Cleavage of structural proteins during the assembly of the head of bacteriophage T4. Nature $1970 \mathbf{2 2 7} 680-685$.

34 Leong MM \& Fox GR. Luminescent detection of immunodot and western blots. Methods in Enzymology 1990184 442-451.

35 Gilden TA \& Wadden TA. The evolution of very-low-calorie diets: an update and meta-analysis. Obesity $2006 \mathbf{1 4} 1283-1293$.

36 Larson-Meyer DE, Heilbronn LK, Redman LM, Newcomer BR, Frisard MI, Anton S, Smith SR, Alfonso A \& Ravussin E. Effect of calorie restriction with or without exercise on insulin sensitivity, beta-cell function, fat cell size, and ectopic lipid in overweight subjects. Diabetes Care 200629 1337-1344.

37 Klein S, Fontana L, Young VL, Coggan AR, Kilo C, Patterson BW \& Mohammed BS. Absence of an effect of liposuction on insulin action and risk factors for coronary heart disease. New England Journal of Medicine $20043 \mathbf{5 0} 2549-2557$.

38 Abbasi F, Lamendola C, McLaughlin T, Hayden J, Reaven GM \& Reaven PD. Plasma adiponectin concentrations do not increase in association with moderate weight loss in insulin-resistant, obese women. Metabolism 200453 280-283.

39 Kotidis EV, Koliakos G, Papavramidis TS \& Papavramidis ST. The effect of biliopancreatic diversion with pylorus-preserving sleeve gastrectomy and duodenal switch on fasting serum ghrelin, leptin and adiponectin levels: is there a hormonal contribution to the weight-reducing effect of this procedure? Obesity Surgery 200616 $554-559$. 
40 Serra A, Granada ML, Romero R, Bayes B, Canton A, Bonet J, Rull M, Alastrue A \& Formiguera X. The effect of bariatric surgery on adipocytokines, renal parameters and other cardiovascular risk factors in severe and very severe obesity: 1-year follow-up. Clinical Nutrition 200625 400-408.

41 Yang WS, Lee WJ, Funahashi T, Tanaka S, Matsuzawa Y, Chao CL, Chen CL, Tai TY \& Chuang LM. Weight reduction increases plasma levels of an adipose-derived anti-inflammatory protein, adiponectin. Journal of Clinical Endocrinology and Metabolism 200186 3815-3819.

42 Lazzer S, Vermorel M, Montaurier C, Meyer M \& Boirie Y. Changes in adipocyte hormones and lipid oxidation associated with weight loss and regain in severely obese adolescents. International Journal of Obesity 200529 1184-1191.

43 Kobayashi H, Ouchi N, Kihara S, Walsh K, Kumada M, Abe Y, Funahashi T \& Matsuzawa Y. Selective suppression of endothelial cell apoptosis by the high molecular weight form of adiponectin. Circulation Research 200494 e27-e31.

44 Ebinuma H, Miyazaki O, Yago H, Hara K, Yamauchi T \& Kadowaki T. A novel ELISA system for selective measurement of human adiponectin multimers by using proteases. Clinica Chimica Acta 2006372 47-53.

45 Garaulet M, Viguerie N, Porubsky S, Klimcakova E, Clement K, Langin D \& Stich V. Adiponectin gene expression and plasma values in obese women during very-low-calorie diet. Relationship with cardiovascular risk factors and insulin resistance. Journal of Clinical Endocrinology and Metabolism 200489 756-760.

46 Klimcakova E, Polak J, Moro C, Hejnova J, Majercik M, Viguerie N, Berlan M, Langin D \& Stich V. Dynamic strength training improves insulin sensitivity without altering plasma levels and gene expression of adipokines in subcutaneous adipose tissue in obese men. Journal of Clinical Endocrinology and Metabolism 200691 5107-5112.

47 Ryan AS \& Nicklas BJ. Reductions in plasma cytokine levels with weight loss improve insulin sensitivity in overweight and obese postmenopausal women. Diabetes Care 200427 1699-1705.
48 Ladeia AM, Adan L, Couto-Silva AC, Hiltner A \& Guimaraes AC. Lipid profile correlates with glycemic control in young patients with type 1 diabetes mellitus. Preventive Cardiology 2006 9 82-88.

49 McLaughlin T, Abbasi F, Cheal K, Chu J, Lamendola C \& Reaven G. Use of metabolic markers to identify overweight individuals who are insulin resistant. Annals of Internal Medicine 2003139 802-809.

50 Bastard JP, Jardel C, Bruckert E, Blondy P, Capeau J, Laville M, Vidal H \& Hainque B. Elevated levels of interleukin 6 are reduced in serum and subcutaneous adipose tissue of obese women after weight loss. Journal of Clinical Endocrinology and Metabolism 2000 85 3338-3342.

51 Wing RR, Blair EH, Bononi P, Marcus MD, Watanabe R \& Bergman RN. Caloric restriction per se is a significant factor in improvements in glycemic control and insulin sensitivity during weight loss in obese NIDDM patients. Diabetes Care 1994 17 30-36.

52 Bodles A, Banga A, Rasouli N, Ono F, Kern PA \& Owens RJ. Pioglitazone increases secretion of high molecular weight adiponectin from adipocytes. American Journal of Physiology. Endocrinology and Metabolism 2006291 E1100-E1105.

53 Polak J, Klimcakova E, Moro C, Viguerie N, Berlan M, Hejnova J, Richterova B, Kraus I, Langin D \& Stich V. Effect of aerobic training on plasma levels and subcutaneous abdominal adipose tissue gene expression of adiponectin, leptin, interleukin 6, and tumor necrosis factor alpha in obese women. Metabolism $2006 \mathbf{5 5}$ 1375-1381.

54 Mather KJ, Hunt AE, Steinberg HO, Paradisi G, Hook G, Katz A, Quon MJ \& Baron AD. Repeatability characteristics of simple indices of insulin resistance: implications for research applications. Journal of Clinical Endocrinology and Metabolism 200186 5457-5464.

Received 12 November 2007

Accepted 30 December 2007 